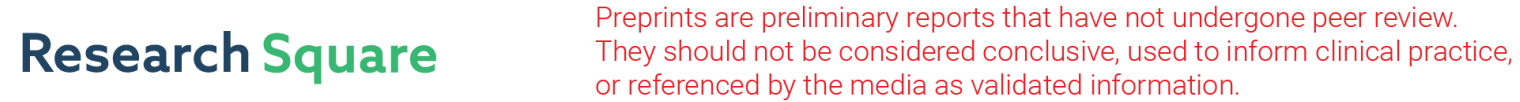 \\ Adaptive Optics for Reduction of Thermal Blooming Effects by Phase Compensation
}

\section{Shuyun Wu}

Institute of Optics and Electronics Chinese Academy of Sciences

Xi Luo ( $D$ luoxi@ioe.ac.cn )

Institute of Optics and Electronics Chinese Academy of Sciences

\section{Xinyang Li}

Institute of Optics and Electronics Chinese Academy of Sciences

\section{Research}

Keywords: Thermal blooming, Adaptive optics, Phase compensation

Posted Date: June 10th, 2020

DOI: https://doi.org/10.21203/rs.3.rs-33690/v1

License: (c) (1) This work is licensed under a Creative Commons Attribution 4.0 International License.

Read Full License

Version of Record: A version of this preprint was published at Journal of Russian Laser Research on July 1st, 2020. See the published version at https://doi.org/10.1007/s10946-020-09894-6. 


\title{
Adaptive Optics for Reduction of Thermal Blooming Effects by \\ Phase Compensation
}

Shuyun Wu, ${ }^{1,2,3}$ Xi Luo, ${ }^{1,2, *}$, Xinyang $\mathrm{Li}^{1,2}$

${ }^{1}$ Key Laboratory on Adaptive Optics, Chinese Academy of Sciences, Chengdu 610209, China

${ }^{2}$ Institute of Optics and Electronics, Chinese Academy of Sciences, Chengdu 610209, China

${ }^{3}$ University of Chinese Academy of Sciences, Beijing 100049, China

corresponding author: luoxi@ioe.ac.cn

first author: wushuyun1993@hotmail.com

\begin{abstract}
The use of an AO system for the reduction of thermal blooming effects by phase correction of a 1.064- $\mu \mathrm{m}$ laser was studied. The energy concentration of the beam spot in the far-field increased greatly when the adaptive optics system performed in a closed loop. The phase compensation of the AO system was effective for a BradleyHermann distortion number of less than 130. The experimental results were in good agreement with the simulation results. This study provides many physical explanations and important conclusions for using adaptive optics to reduce the thermal blooming effect.
\end{abstract}

Keywords: Thermal blooming; Adaptive optics; Phase compensation

\section{Introduction}

Heat-induced changes in the optical parameters of a medium leads to changes in radiation propagation of high energy laser (HEL) beams. This change is referred to as “thermal blooming" [1-6]. Thermal blooming causes a negative-lens-like optical effect in the atmosphere that blooms energy out of the beam. This phenomenon can defocus, scatter and distort the optical beam [7]. An adaptive optics (AO) system corrects the effects of thermal blooming by applying a positive-lens-like compensation to the HEL beam. In theory, by focusing the HEL beam, this should override the blooming.

Bradley and Herrmann investigated the use of AO systems to mitigate nonlinear optical effects induced by thermal blooming. They demonstrated an improvement in the system performance with phase compensation of thermal blooming [8]. Since then, several research institutions have studied HEL propagation in the atmosphere and its 
phase correction, such as MIT Lincoln Laboratory [9-10], Lawrence Livermore National Laboratory [11], and the Air Force Weapons Laboratory [12]. However, there are few experimental reports on the phase compensation of thermal blooming by AO system.

The purpose of this research is to demonstrate the use of an AO system to correct the phase of a collimated Gaussian beam and reduce thermal blooming. The AO system was also used to increase the peak far-field irradiance by correcting the phase of aberrations in the optical system used for the blooming experiments.

\section{Theoretical analysis}

The irradiance distribution $I(x, y)$ of a collimated Gaussian beam is expressed as

$$
I(x, y)=\left(2 P / \pi w^{2}\right) \times \exp \left[-2\left(x^{2}+y^{2}\right) / w^{2}\right]
$$

where $P$ is the initial beam power, and $D=2 w$ is the $1 / e^{2}$ intensity beam diameter. The phase distortion $\varphi_{B}$ accumulated along the propagation path $L$ and induced by steadystate thermal blooming, which was assumed to be a constant absorption coefficient, $\alpha$, and a transverse wind speed, $V$ (such as along $\mathrm{X}$-axis positive direction), is given by

$$
\varphi_{B}(x, y)=n_{T} \times \frac{\alpha}{\rho_{0} C_{p}} \times \frac{k L}{V} \int_{-\infty}^{x} I\left(x^{\prime}, y\right) d x^{\prime},
$$

where $n_{T}=d n / d T$ is the change in refractive index with respect to the temperature of medium at constant pressure, and $\rho_{0}, k$, and $C_{p}$, are the density, wave number and specific heat at constant pressure, respectively [13].

By substituting Eq. (1) into Eq. (2), the blooming phase $\varphi_{G B}$ is obtained as

$$
\varphi_{B G}=-\frac{\Delta \varphi_{G}}{2} \exp \left[-2\left(\frac{y}{w}\right)^{2}\right]\left[1+\operatorname{erf}\left(\frac{\sqrt{2} x}{w}\right)\right] .
$$


The maximum phase shift is

$$
\Delta \varphi_{G}=(1 / 2 \sqrt{\pi}) N_{D}
$$

where

$$
N_{D}=\frac{4 \sqrt{2}\left(-n_{T}\right) k \alpha P L}{\rho_{0} C_{p} V D}
$$

is the Bradley-Hermann distortion number commonly used as a measure of the strength of thermal blooming [13]. Phase distortion information can be obtained by the ShackHartmann wave-front sensor in an open-loop adaptive optics system. Using Eq. (5) to obtain the Bradley-Hermann thermal distortion parameter, $N_{D}$, of the experiment.

\section{Numerical calculation results and analysis}

The thermal blooming effect of laser beams propagating through horizontal atmosphere was studied by performing numerical simulations on Easy-Laser software [14]. In particular, the phase screen simulation method was based on a procedure that replaced a continuous medium of radiation by a sequence of thin screens, simulating distortions acquired by the optical wave through its propagation [15]. Figure 1 shows a simulation of the AO system correcting thermal blooming by applying phase compensations. 


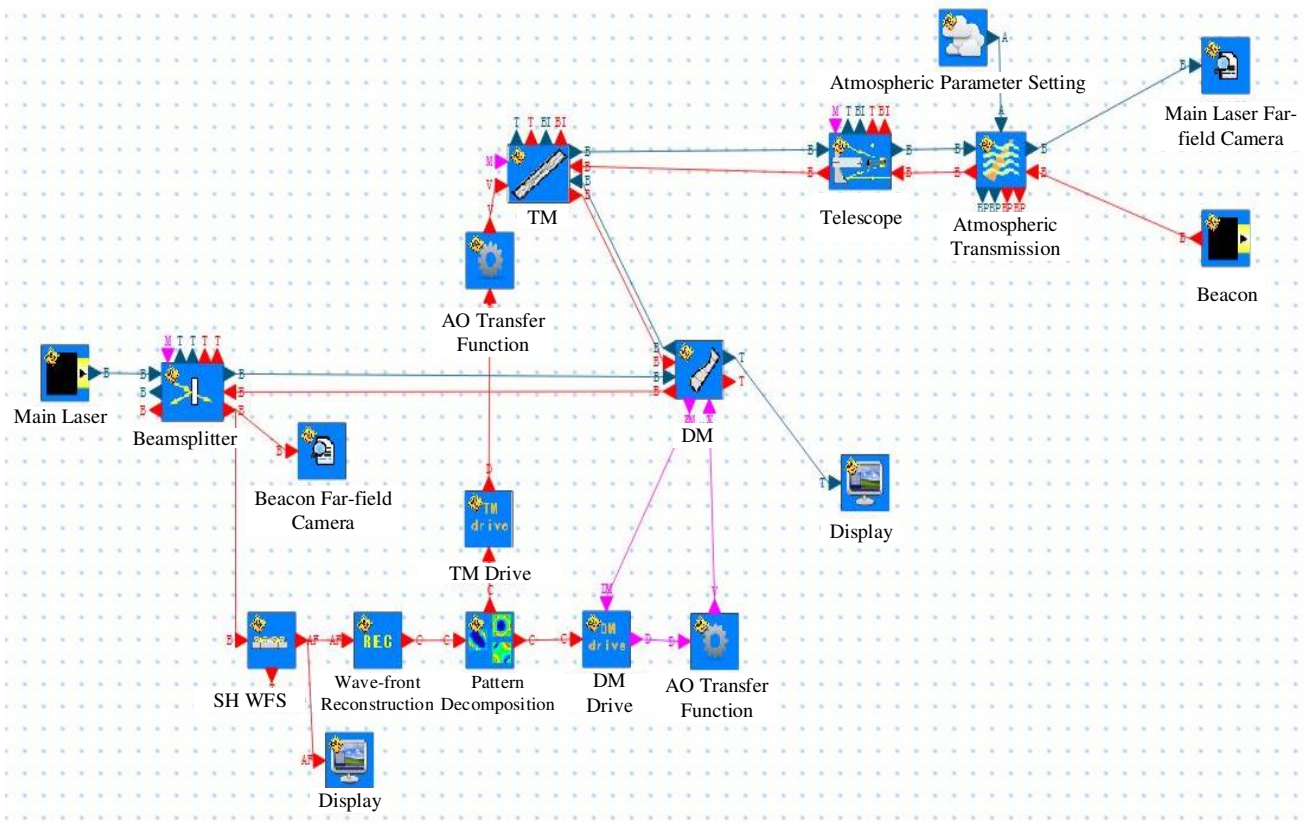

Fig. 1. Schematic diagram of the AO system corrects the thermal blooming by applying the phase compensations in software.

For the simulation, we used an AO system that performed phase compensation using a Shack-Hartmann (SH) wave-front sensor (WFS), a tilt mirror (TM), and a deformable mirror (DM) with 127 actuators. Figure 2 shows a schematic of the AO system for phase compensation of the thermal blooming setup.

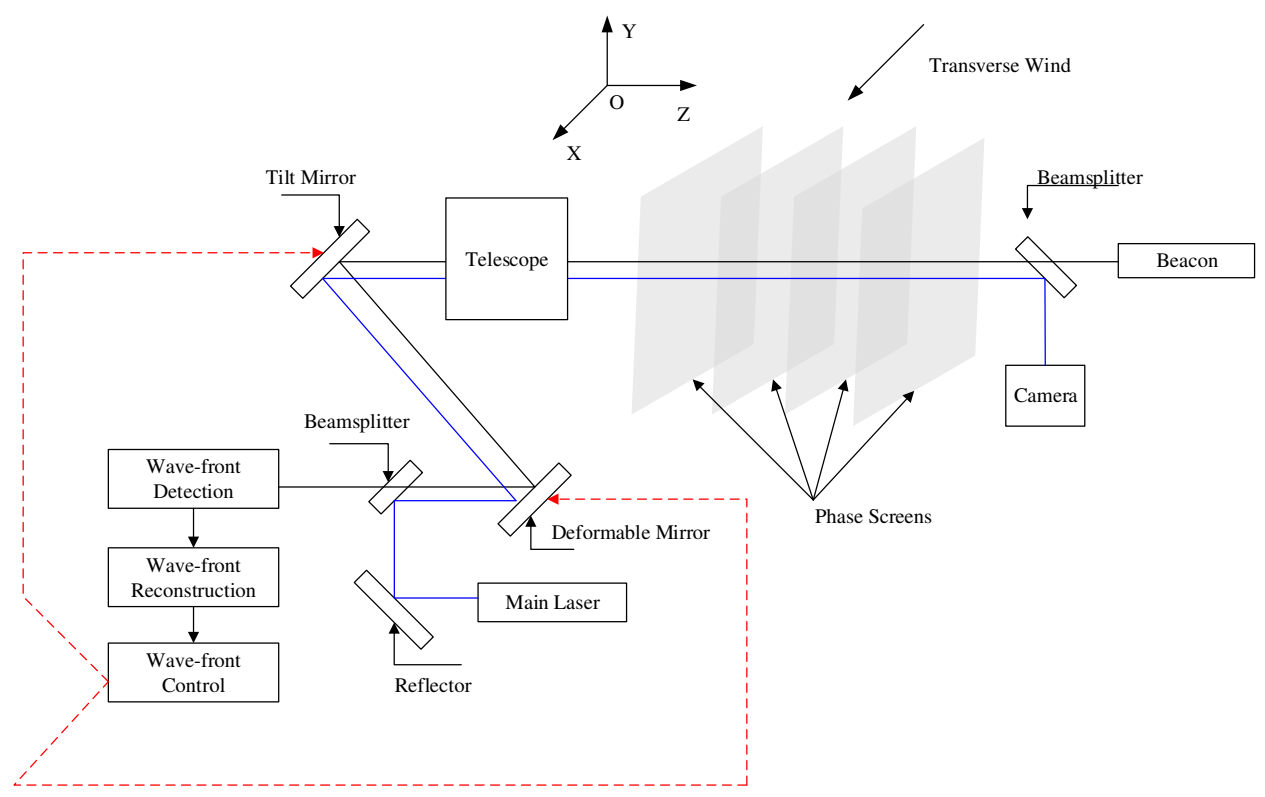

Fig. 2. Schematic of the AO system for phase compensation of thermal blooming. 
In this scenario, a beacon signal was transmitted from the target plane to the source plane with the effects of current phase screen due to thermal blooming. The phase of the beacon in the source plane was then applied directly to the HEL beam, and the idealized phase compensation process was repeated.

Table 1 presents the parameters used in the simulation.

Table 1 Simulation parameters

\begin{tabular}{|c|c|}
\hline Simulation & Set up \\
\hline Effective aperture of SH WFS (mm) & 148 \\
\hline SH WFS subapertures size (Relay optical path) (mm) & 12.33 \\
\hline Layout of SH WFS subapertures & $12 \times 12$ \\
\hline SH WFS subaperture pixel & 20 \\
\hline SH WFS subaperture single pixel field angle (relay optical path) ( $\mu \mathrm{rad})$ & 24.5 \\
\hline Number of deformable mirror actuator & 127 \\
\hline $\begin{array}{l}\text { Distance between deformable mirror actuators (relay optical path) } \\
\qquad(\mathrm{mm})\end{array}$ & 12.2 \\
\hline System sampling frequency $(\mathrm{Hz})$ & 1,000 \\
\hline Simulated laser distribution & $\begin{array}{l}\text { Collimated Gaussian } \\
\text { beam }\end{array}$ \\
\hline Simulated beacon distribution & Plane wave beam \\
\hline Simulated laser wavelength $(\mu \mathrm{m})$ & 1.064 \\
\hline Simulated beacon wavelength $(\mu \mathrm{m})$ & 0.532 \\
\hline Beacon beam diameter (Relay optical path) (mm) & 148 \\
\hline Telescope magnification & 5 \\
\hline Telescope focal length & Infinity \\
\hline $\begin{array}{l}\text { Transmission aperture of collimated beam in horizontal atmosphere } \\
\qquad(\mathrm{mm})\end{array}$ & 740 \\
\hline $\begin{array}{l}\text { Transmission distance of collimated beam in horizontal atmosphere } \\
\qquad(\mathrm{km})\end{array}$ & 2 \\
\hline Absorption coefficient $(1 / \mathrm{km})$ & 0.07 \\
\hline Transverse wind speed $(\mathrm{m} / \mathrm{s})$ & 2 \\
\hline Density $\left(\mathrm{kg} / \mathrm{m}^{3}\right)$ & 1.213 \\
\hline Specific heat at constant pressure (J/kg.K) & 1,006 \\
\hline
\end{tabular}


In the simulations, the Bradley-Hermann distortion parameter $\left(N_{D}\right)$ can be changed by adjusting the laser's power (from $3000 \mathrm{~W}$ to $39,000 \mathrm{~W}$ ). A quantitative assessment of the relative power-in-the-bucket (PIB) was performed to analyze the far-field spot. The most common metric for gauging the performance of HEL systems is PIB, the power within a circular region in the cross section of the laser beam, which is situated at the laser aimpoint on the target [16]. The higher the distortion parameter, the likelier the increase in bucket size. Comparison of bucket radii $(\mathrm{PIB}=63.2 \%)$ in far-field (equivalent focal length $=3,365.992 \mathrm{~m}$ ) with different Bradley-Hermann distortion numbers $\left(N_{D}=10,20,30,50,70,100,120\right.$ and 130) between open-loop and closedloop AO systems is given in Fig. 3. 

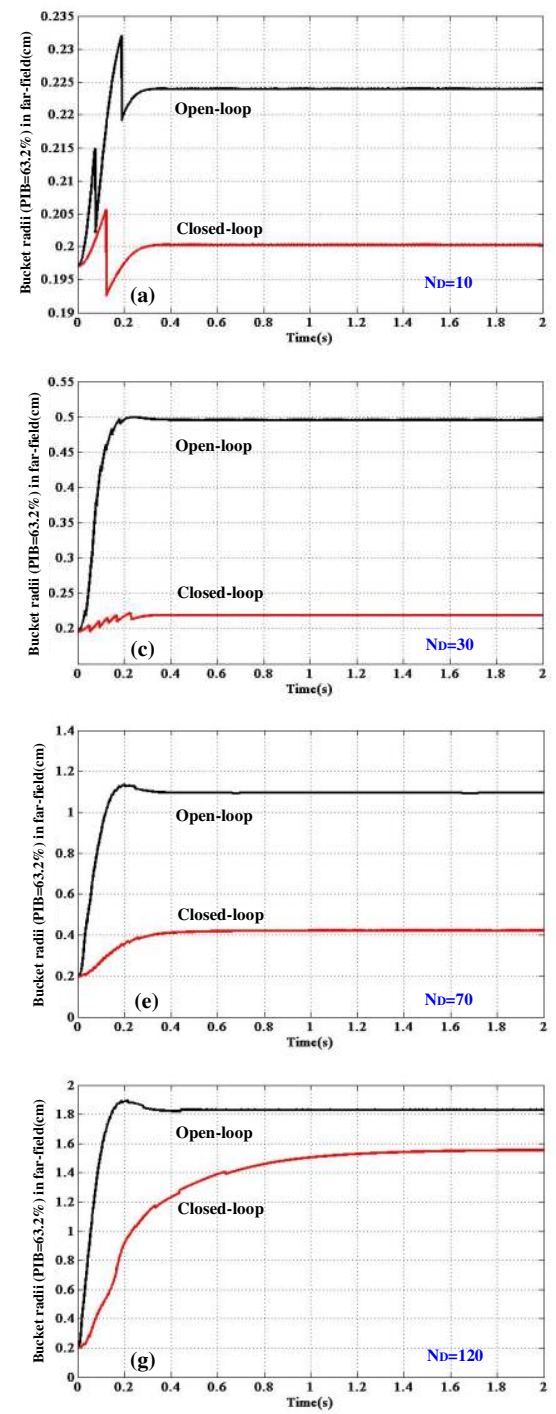
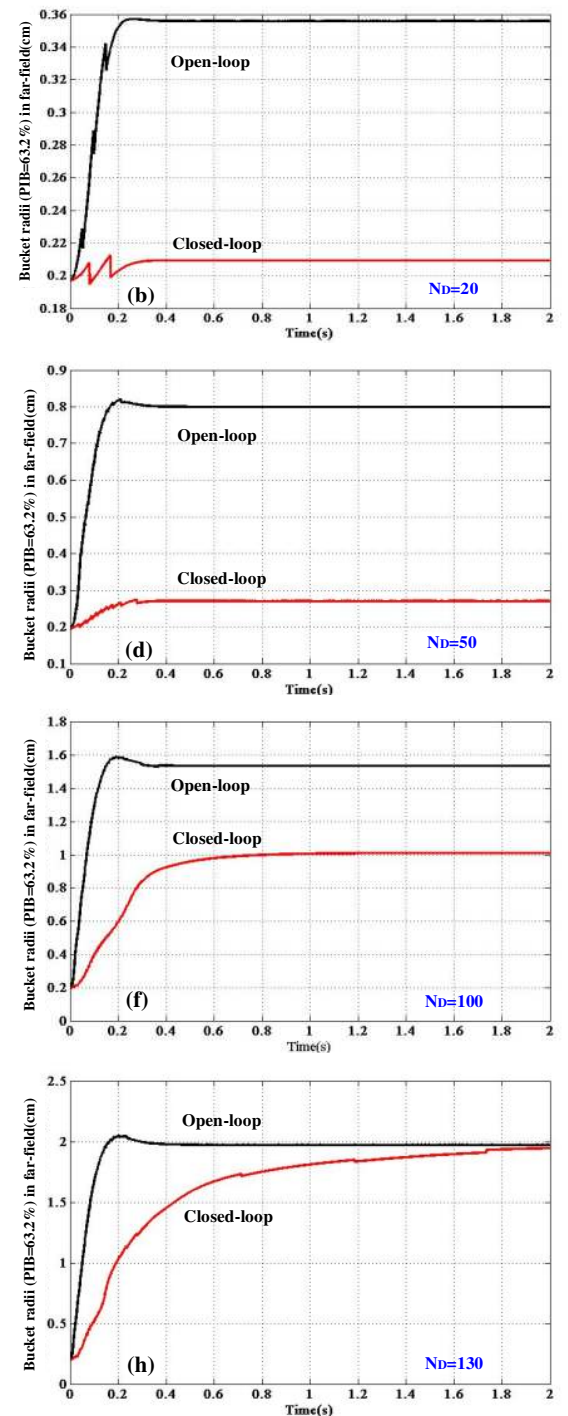

Fig. 3. Comparison of bucket radii $(\mathrm{PIB}=63.2 \%)$ in far-field with different Bradley-Hermann distortion numbers $\left(N_{D}=10,20,30,50,70,100,120\right.$ and 130) of open-loop and closed-loop AO systems.

Figure 4 shows the bucket radii $(\mathrm{PIB}=63.2 \%)$ in far-field under the different Bradley-Hermann distortion numbers with open- and closed-loop operations of the AO system. 


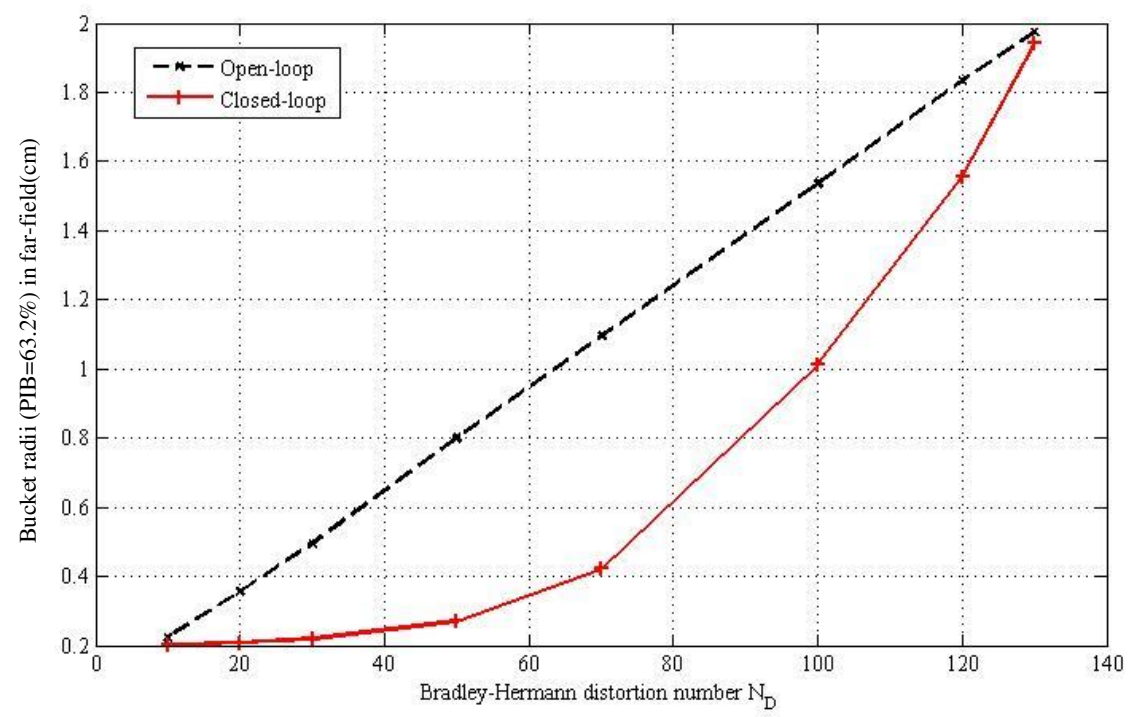

Fig. 4 Bucket radii $(\mathrm{PIB}=63.2 \%)$ in far-field with open- and closed-loop operations of the AO system vs. the Bradley-Hermann distortion number $\left(N_{D}=10,20,30,50,70,100,120\right.$ and 130)

Then, the magnification of beam quality factor $\beta$ is used to evaluate the ability of phase compensation of the AO system and reduce the thermal blooming effect. This evaluation method can effectively reduce system aberrations. $\beta$ is defined as

$$
\beta=\frac{\theta_{\text {REAL-PIB }}}{\theta_{I D E A L-P I B}},
$$

where $\theta_{\text {REAL-PIB }}$ and $\theta_{I D E A L-P I B}$ are the bucket sizes in far-field with specific powers of real and ideal scenarios, respectively [17].

Since $\theta_{I D E A L-P I B}$ is independent of the open/closed loop of the AO system, the magnification of $\beta$ is expressed as

$$
\beta_{\text {Times }}=\frac{\left(\theta_{\text {REAL-PIB }}\right)_{\text {Open-loop }}}{\left(\theta_{\text {REAL-PIB }}\right)_{\text {Close-loop }}} .
$$

In this equation, $\beta_{\text {Times }}>1$ means that compensation of the $\mathrm{AO}$ system influences the thermal blooming effect. The higher the $\beta_{\text {Times }}$, the greater the influence.

The different values of $\beta_{\text {Times }}$ as a function of $N_{D}$ are shown in Fig. 5. 


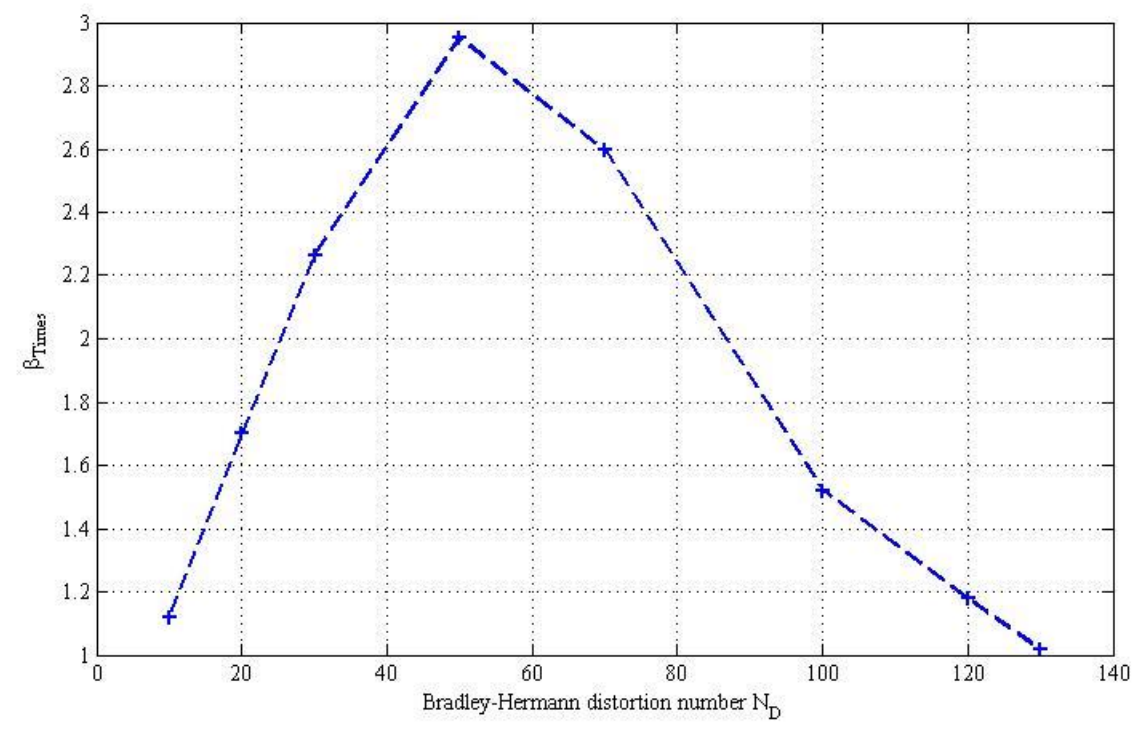

Fig. 5. $\beta_{\text {Times }}$ vs. $N_{D}$

The simulation results from Fig. 5 reveal that the thermal blooming effect for a collimated Gaussian beam propagating through uniform wind can be significantly reduced by using the $\mathrm{AO}$ system to make the appropriate phase compensation. The accumulation of heat at the beginning of the curve is weak, the influence of thermal blooming on the shape of the far-field spot is not severe, and the effect of phase compensation is not particularly obvious. An important characteristic of the thermal blooming process is its effect on limiting the peak irradiance, independent of the power available at the transmitter. The AO system mentioned above is effective in reducing the thermal blooming in the range of $N_{D}=10-130$. Unfortunately, the HEL beam only concentrates the energy contained within the beam and further heats up the surrounding atmosphere. The AO system attempts to correct for the increased blooming with even higher positive-lens-like compensation, and the process reinforces itself, ultimately causing failure of the AO system through thermal accumulation.

\section{Experimental results and analysis}


It is difficult to setup a full-scale experiment to investigate phase compensation of thermal blooming. Such an experiment would require an HEL system, an AO system robust enough to handle the HEL beam powers needed for thermal blooming, a test range, and a facility large enough to conduct the experiment. Therefore, from the perspective of feasibility, in this experiment, we used $\mathrm{CH}_{3} \mathrm{CH}_{2} \mathrm{OH}$ as the absorption medium and a low-power laser to generate severe thermal blooming. The experimental arrangement is illustrated in Fig. 6. A photograph of the experimental layout is illustrated in Fig. 7.

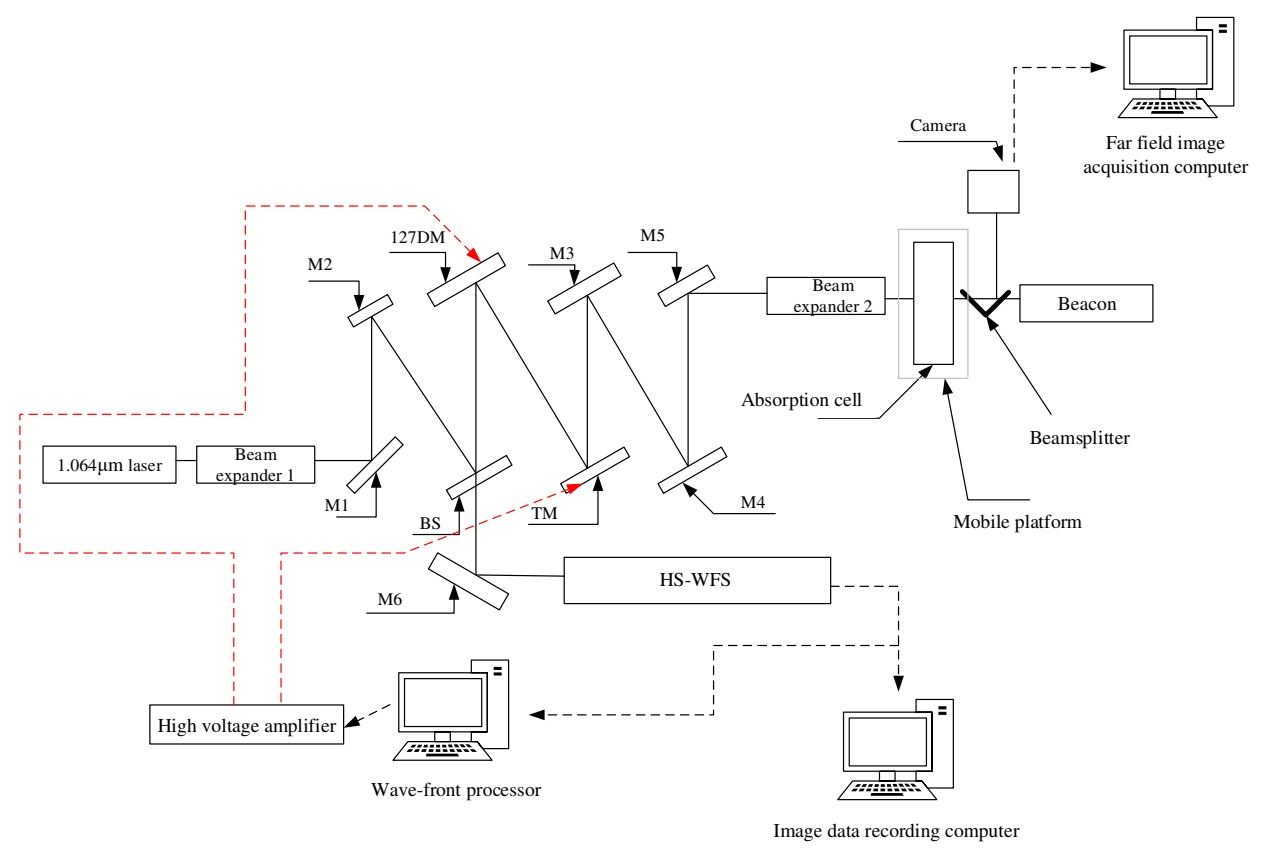

Fig. 6. Schematic of experiment with AO system for phase compensation of thermal blooming. 


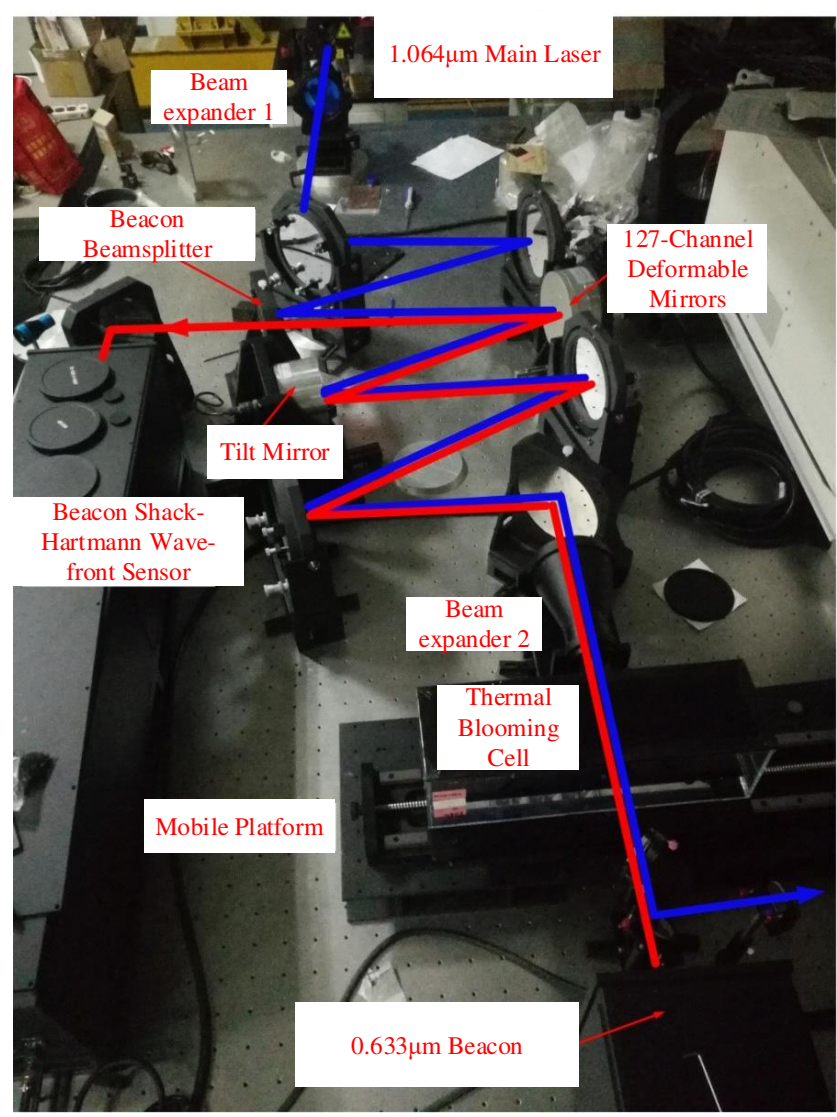

Fig. 7. Photograph of experimental layout.

Parameters of the system that were used in the experiment are presented in Table 2.

Table 2 Experimental parameters of $\mathrm{CH}_{3} \mathrm{CH}_{2} \mathrm{OH}$ as the absorption medium

\begin{tabular}{cc}
\hline Experiment & Parameters \\
\hline Effective wavelength of SH WFS $(\mu \mathrm{m})$ & 0.633 \\
SH WFS subapertures size (Clear aperture) $(\mathrm{mm})$ & $10 \times 10$ \\
Layout of SH WFS subapertures & $12 \times 12$ \\
SH WFS sampling frequency $(\mathrm{Hz})$ & 2,500 \\
Number of DM actuators & \pm 3 \\
DM actuators delivering the mechanical stroke $(\mu \mathrm{m})$ & 127 \\
Distance between DM actuators (Relay optical path) $(\mathrm{mm})$ & 12.2 \\
Effective wavelength of DM $(\mu \mathrm{m})$ & 0.633 and 1.064 \\
Experimental laser distribution & Collimated Gaussian beam \\
Experimental beacon distribution & Plane wave beam \\
Experimental laser wavelength $(\mu \mathrm{m})$ & 1.064 \\
Experimental beacon wavelength $(\mu \mathrm{m})$ & 0.633 \\
Laser power $(\mathrm{w})$ & $1-3$
\end{tabular}


Absorption coefficient of ethanol $\left(\mathrm{cm}^{-1}\right)$

The length of the thermal blooming cell $(\mathrm{cm})$

Moving speed of the thermal blooming cell $(\mathrm{mm} / \mathrm{s})$

Beam diameter in the thermal blooming cell ( $\mathrm{mm})$
$0.11[18]$

15

$1-20$

8

Layout of deformable mirror with 127 actuators is shown in Fig. 8.

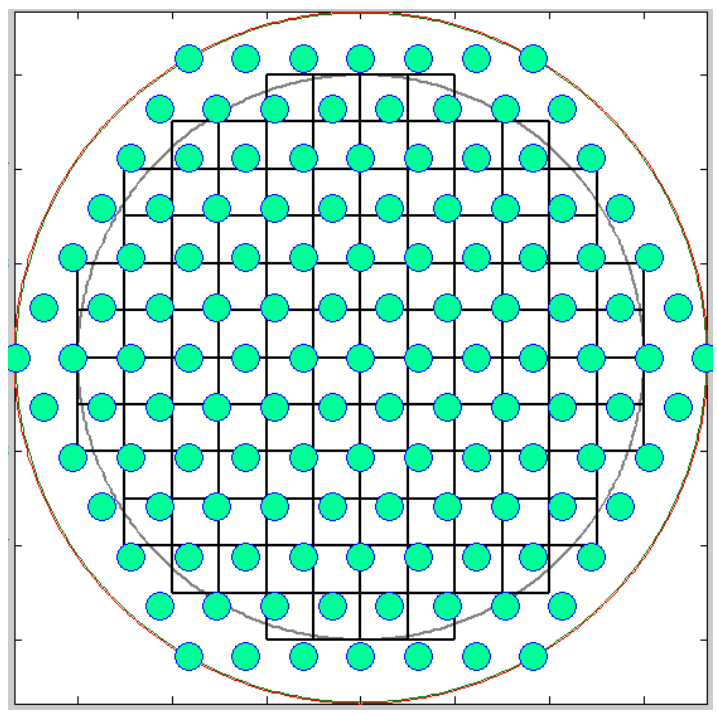

Fig. 8. Schematic of deformable mirror with 127 actuators.

It should be noted that the theoretical range of $N_{D}$ that can be generated by a thermal blooming cell in the above scenario is approximately from 37 to 410 .

In the experiment, the transverse wind speed, V (from $1 \mathrm{~mm} / \mathrm{s}$ to $20 \mathrm{~mm} / \mathrm{s}$ ), was simulated by transversing the platform [19]. A vast difference between the open- and closed-loop operations of the AO system can be observed. Figure 9 shows the results of the experiment with wave-front errors PV/RMS for the AO system's operations for cases where $\mathrm{V}$ was $5 \mathrm{~mm} / \mathrm{s}, 10 \mathrm{~mm} / \mathrm{s}$ and $15 \mathrm{~mm} / \mathrm{s}$, respectively. 

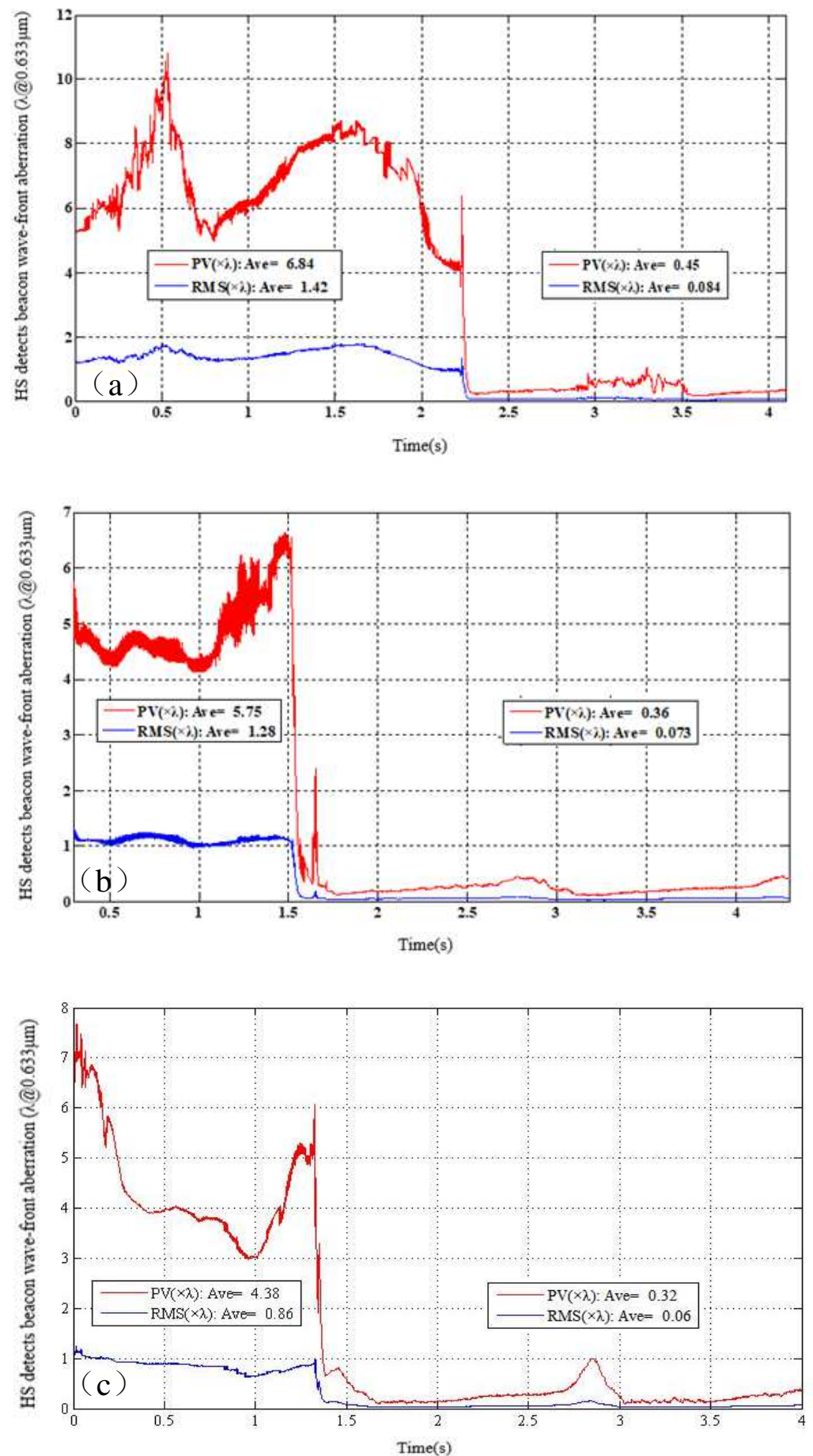

Fig. 9. Comparison of wave-front PV/RMS between open-loop and closed-loop AO systems. In (a) platform velocity $V$ is $5 \mathrm{~mm} / \mathrm{s}$; wave-front in open-loop AO system, $\mathrm{PV}=6.84 \lambda$ and $\mathrm{RMS}=1.42 \lambda$; wave-front in closed-loop AO system, $\mathrm{PV}=0.45 \lambda$ and $\mathrm{RMS}=0.084 \lambda$, in (b) platform moving velocity

$V$ is $10 \mathrm{~mm} / \mathrm{s}$; wave-front in open-loop AO system, $\mathrm{PV}=5.75 \lambda$ and $\mathrm{RMS}=1.28 \lambda$; wave-front in closed-loop AO system, $\mathrm{PV}=0.36 \lambda$ and $\mathrm{RMS}=0.073 \lambda$ and in (c) platform moving velocity $V$ is 15 $\mathrm{mm} / \mathrm{s}$; wave-front in open-loop AO system, PV $=4.38 \lambda$ and $\mathrm{RMS}=0.86 \lambda$; wave-front in closed-loop $\mathrm{AO}$ system, $\mathrm{PV}=0.32 \lambda$ and $\mathrm{RMS}=0.06 \lambda$.

In Fig. 9, a significant decrease in wave-front errors can be observed after phase 
compensation of the closed-loop operation of the AO system for thermal blooming. It should be noted that the wave-front phase information provided by SH WSF while the AO system was performing the open-loop operation can deduce $N_{D}$ from Eq. (4). In these three scenarios, the $N_{D} S$ are 82.18, 74.08 and 49.27, respectively. Typical results of far-field images are shown in Fig. 10.
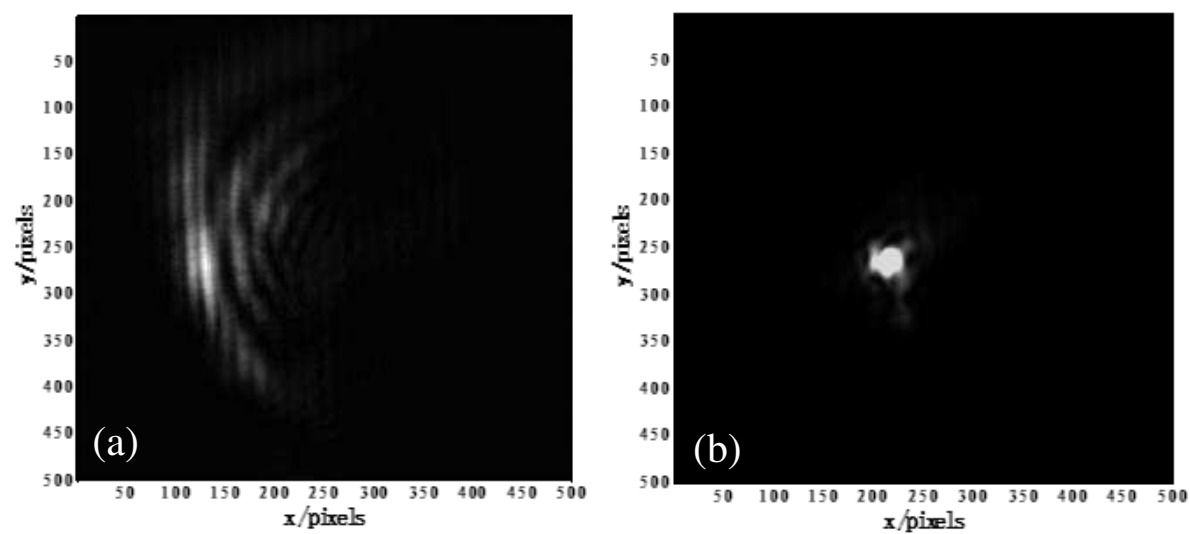

Fig.10. Far-field images of main laser (a) with open-loop operation of the AO system and (b) with closed-loop operation of the AO system.

Figure 11 shows the far-field bucket radius $(\mathrm{PIB}=63.2 \%)$ under $\mathrm{N}_{\mathrm{D}}$ before and after compensation of the AO system. 

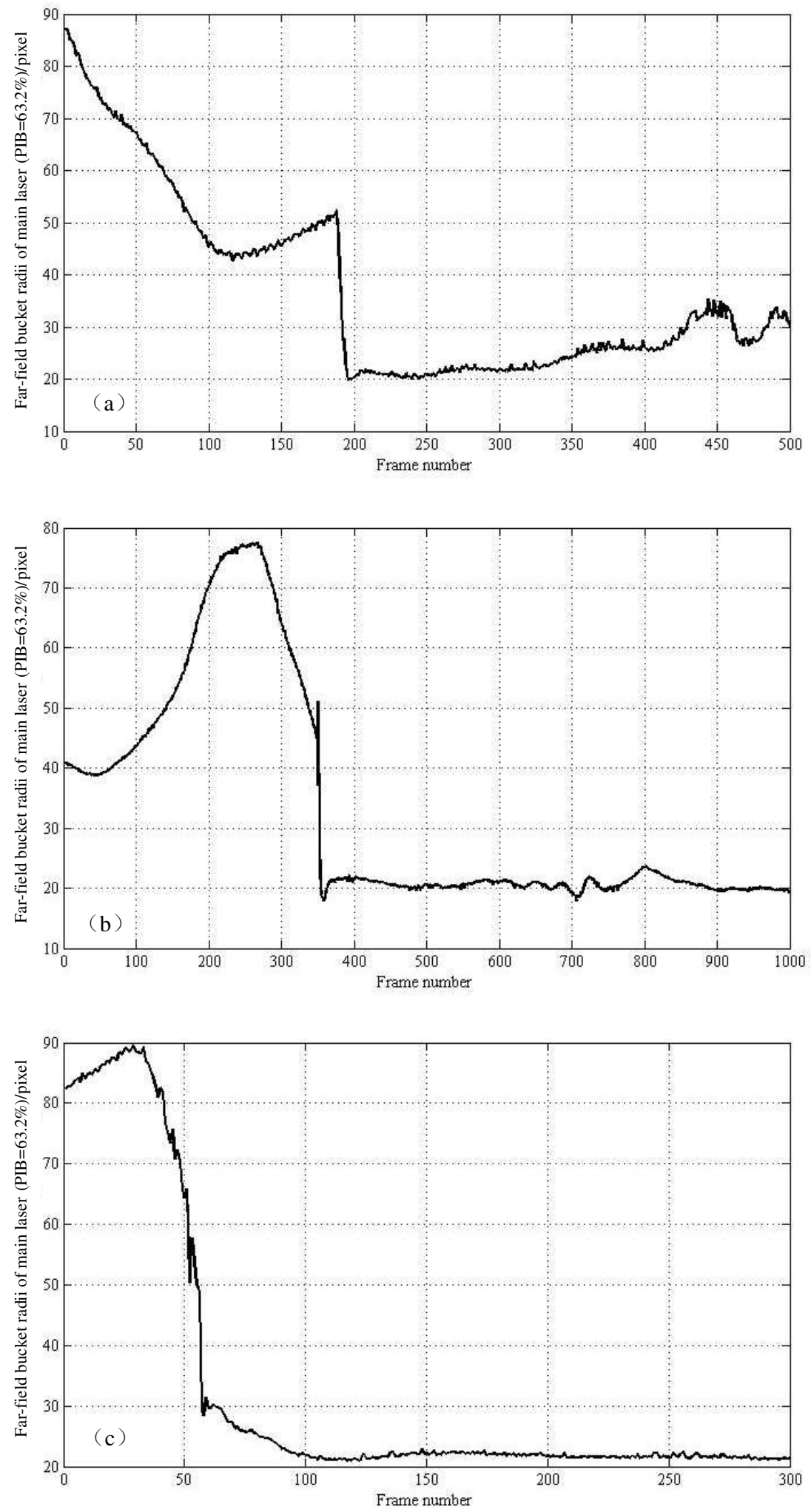

Fig. 11. Comparison of far-field bucket radii $(\mathrm{PIB}=63.2 \%)$ between open- and closed-loop operations of $A O$ system. In (a) $\mathrm{N}_{\mathrm{D}}=82.18$, in (b) $\mathrm{N}_{\mathrm{D}}=74.08$ and in (c) $\mathrm{N}_{\mathrm{D}}=49.27$.

As shown in Fig. 11, phase compensation of the AO system can greatly increase the 
energy concentration of the beam spot in the far-field when $N_{D}$ are $82.18,74.08$, and 49.27, respectively. To quantitatively analyze the effect of phase compensation of the $\mathrm{AO}$ system, $\beta_{\text {Times }}$ under different thermal distortion number conditions have been derived. The $\beta_{\text {Times }}$ are $2.26,2.70$ and 3.59 when $N_{D}$ are $82.18,74.08$ and 49.27, respectively. Therefore, the effect of phase compensation of an AO system on thermal blooming is significant. Figure 12 compares the $\beta_{\text {Times }}$ of simulations with those of the experiments. To obtain the error range, mean value of the far-field bucket radius (PIB $=63.2 \%$ ) with open-loop operation was divided by its maximum and minimum values with the closed-loop operation.

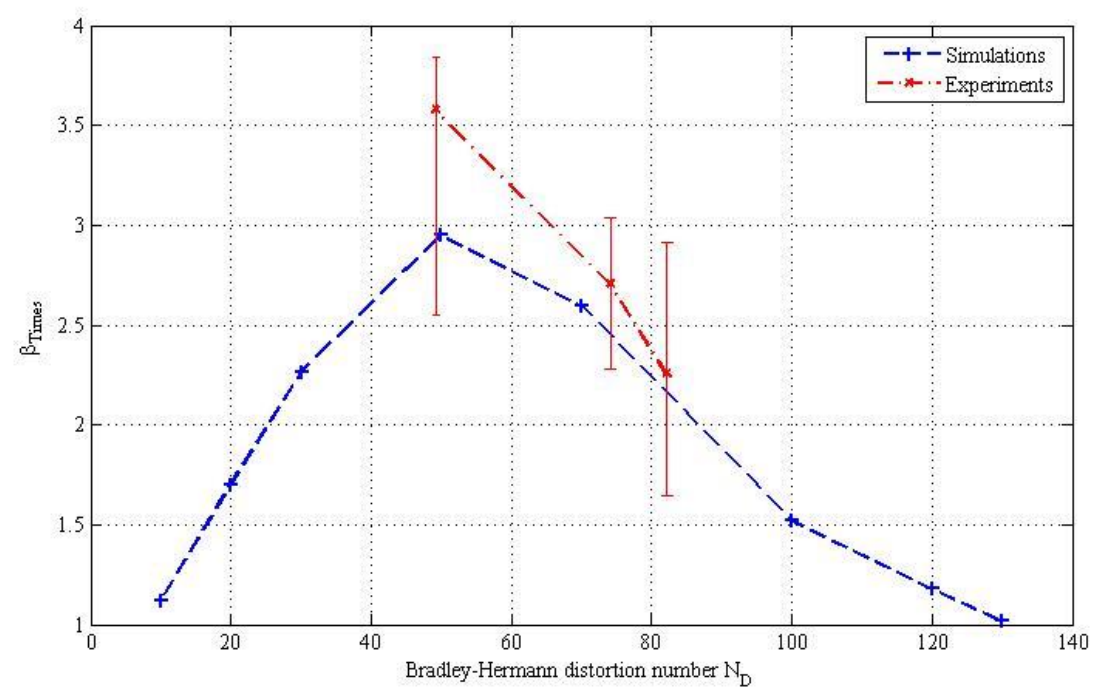

Fig. 12. Comparison of $\beta_{\text {Times }}$ of simulations with experiments.

When the speed of the mobile platform was low, the thermal blooming effect would generate a large amount of accumulated heat, and the range of phase compensation of the AO system is limited. Therefore, the range of $N_{D}$ generated by the experiments were smaller than those generated by the simulations. Hence, the experimental results are consistent with the simulation results. 


\section{Conclusions}

In this paper, phase compensation of $\mathrm{AO}$ system for thermal blooming reduction is studied through simulation and experiment. In the simulation, the AO system was used to compensate thermal blooming phase distortion by $1.064-\mu \mathrm{m}$ laser propagating through the horizontal atmosphere. In the experiment, $\mathrm{CH}_{3} \mathrm{CH}_{2} \mathrm{OH}$ was used as the absorption medium instead of horizontal atmosphere, and transverse wind speed was generated by moving the platform carrying $\mathrm{CH}_{3} \mathrm{CH}_{2} \mathrm{OH}$. Thermal distortion was induced by the low-power laser in the medium. Simulation results show that the closedloop operation of $\mathrm{AO}$ can enhance the phase compensation of thermal blooming when $N_{D}$ is approximately 50 . When $N_{D}$ is less than 130 , the AO system will improve beam quality of the far-field spot. Additionally, experimental results showing beam quality of the far-field was improved, which is consistent with the results obtained numerically. This research can provide a reference value for engineering applications requiring efficient HEL propagation.

\section{List of abbreviations}

HEL: high energy laser; AO: adaptive optics; SH: Shack-Hartmann; WFS: wave-front sensor; TM: tilt mirror; PIB: power-in-the-bucket

\section{Availability of data and materials}

Detail about data has been provided in the manuscript.

\section{Competing interests}

The authors declare that they have no competing interests. 


\section{Funding}

This work was supported by the Chinese Academy of Sciences Innovation Fund [grant number CXJJ-16S022].

\section{Authors' contributions}

SYW and XL carried out the theoretical analysis, numerical simulation and participated in the discussion of experimental results. All authors engaged in the idea of the method. The writing of the manuscript was done by SYW.

\section{Acknowledgements}

Not applicable

\section{References}

1. Wallace, J., Pasciak, J.: Compensating for thermal blooming of repetitively pulsed lasers. J. Opt. Soc. Am. 65(11), 1257-1260 (1975)

2. McLean, E.A., Sica, L., Glass, A.J.: Interferometric observation of absorption induced index change associated with thermal blooming. Appl. Phys. Lett. 13(11), 369-371 (1968)

3. Pearson, J.E., Yeh, C., Brown, W.P: Propagation of laser beams having an on-axis null in the presence of thermal blooming. J. Opt. Soc. Am. 66(12), 1384-1388 (1976)

4. Chan, C.H.: Effective absorption for thermal blooming due to aerosols. Appl. Phys. Lett. 26(11), 628-630 (1975)

5. Bridges, W.B., Pearson, J.E.: Thermal blooming compensation using coherent optical adaptive techniques (COAT). Appl. Phys. Lett. 26(9), 539-543 (1975)

6. Brown, R.T., Smith, D.C.: Aerosol-induced thermal blooming. J. Appl. Phys. 46(1), 402-405 (1975)

7. Gebhardt, F.G.: Experimental demonstration of the use of phase correction to reduce thermal blooming. J. Mod. Optic. 26(5), 615-625 (1979)

8. Bradley, L.C., Herrmann, J.: Phase compensation for thermal blooming. Appl. Opt. 13(2), 331334 (1974)

9. Johnson, B., Schonfeld, J.F.: Demonstration of spontaneous thermal-blooming phasecompensation instability. Opt. Lett. 16(16), 1258-1260 (1991)

10. Schonfeld, J.F.: Linearized theory of thermal-blooming phase-compensation instability with realistic adaptive-optics geometry. J. Opt. Soc. Am. B 9(10), 1803-1812 (1992).

11. Karr, T.J., Morris, J.R., Chambers, D.H., Viecelli, J.A., Cramer, P.G.: Cramer: Perturbation growth by thermal blooming in turbulence. J. Opt. Soc. Am. B 7(6), 1103-1124 (1990) 
12. Wetterer, C.W., Schelonka, L.P. Kramer, M.A.: Correction of thermal blooming by optical phase conjugation. Opt. Lett. 14(16), 874-876 (1989)

13. Gebhardt, F.G.: Twenty-five years of thermal blooming: An Overview. Proc. SPIE 1221, 2-25 (1990)

14. Gang, X., Zhang, J., Luo, W., Fang, Y.: Component Based 3D simulation tool for laser transmission path design. Comput. S. 34(6), 389-393 (2017)

15. Banakh, V.A., Falits, A.V.: Numerical simulation of propagation of laser beams formed by multielement apertures in a turbulent atmosphere under thermal blooming. Atmos. Ocean. Opt. 26(6), 455-465 (2013)

16. Van Zandt, N.R., Fiorino, S.T., Keefer, K.J.: Enhanced, fast-running scaling law model of thermal blooming and turbulence effects on high energy laser propagation. Opt. Express 12(12), 1478914798 (2013)

17. Du, X.: Factor for evaluating beam quality of a real high-power laser on the target surface in far field. Chin. J. Lasers 24(4), 327-332 (1997)

18. Kedenburg, S., Vieweg, M., Gissibl, T., Giessen, H.: Linear refractive index and absorption measurements of nonlinear optical liquids in the visible and near-infrared spectral region. Opt. Mater. Express 2(11), 1588-1611 (2012)

19. Li, Z., Li, X.: Fundamental performance of transverse wind estimator from Shack-Hartmann wave-front sensor measurements. Opt. Express 26(9), 11859-11876 (2018) 


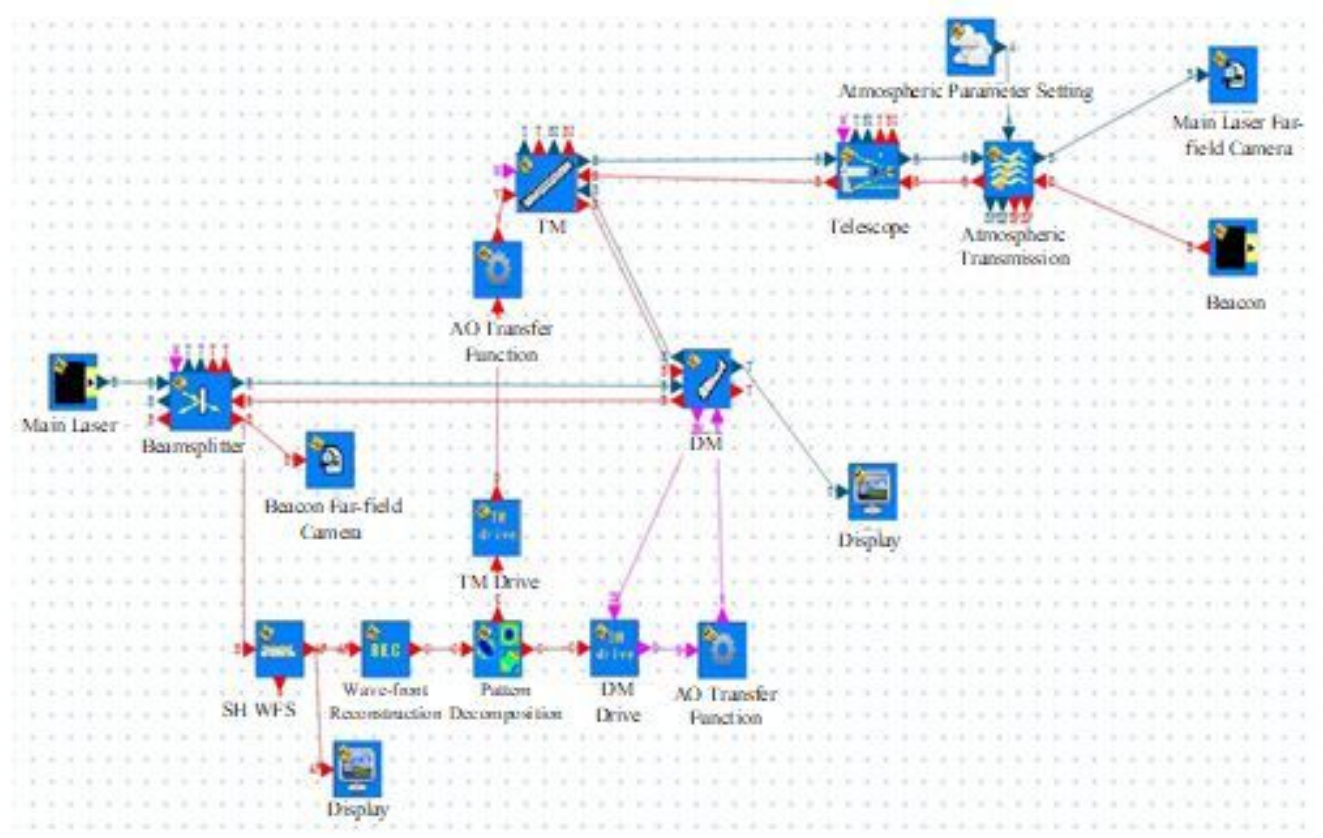

\section{Figure 1}

Schematic diagram of the $\mathrm{AO}$ system corrects the thermal blooming by applying the phase compensations in software.

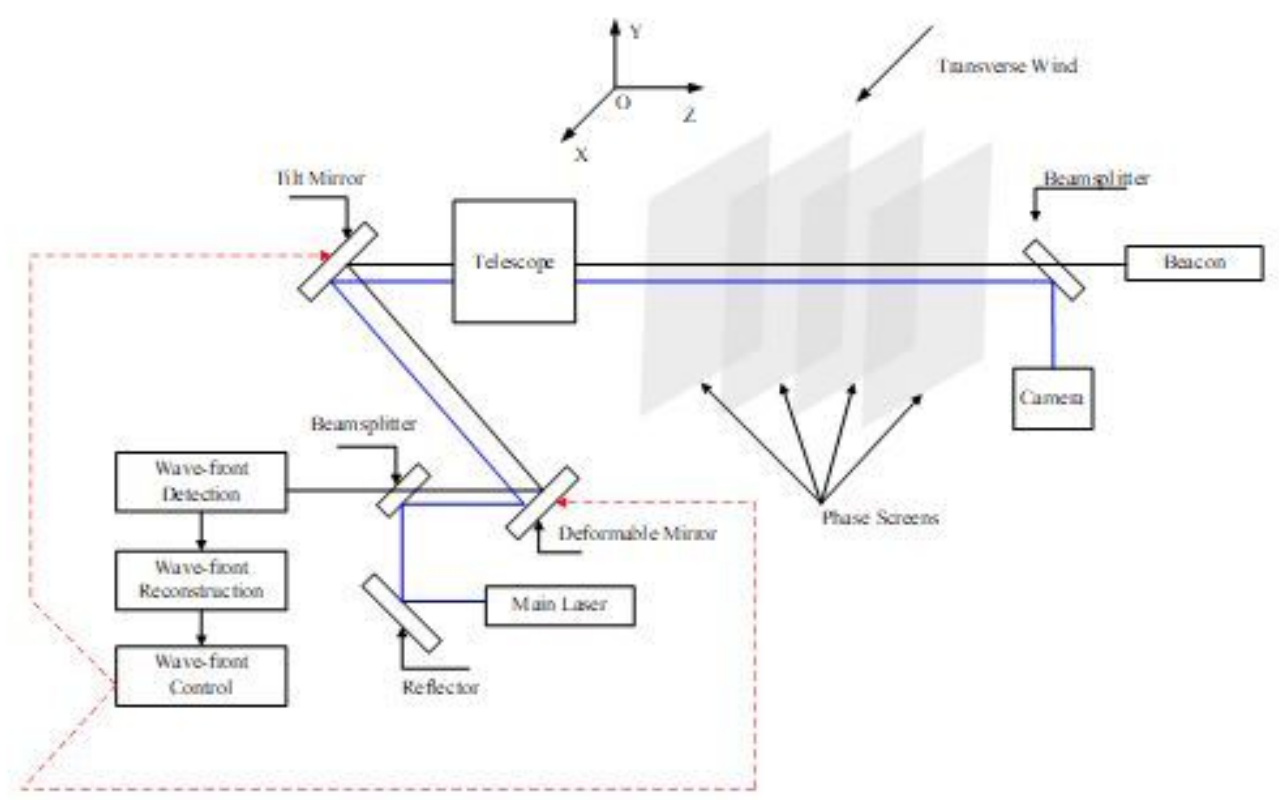

Figure 2

Schematic of the AO system for phase compensation of thermal blooming. 

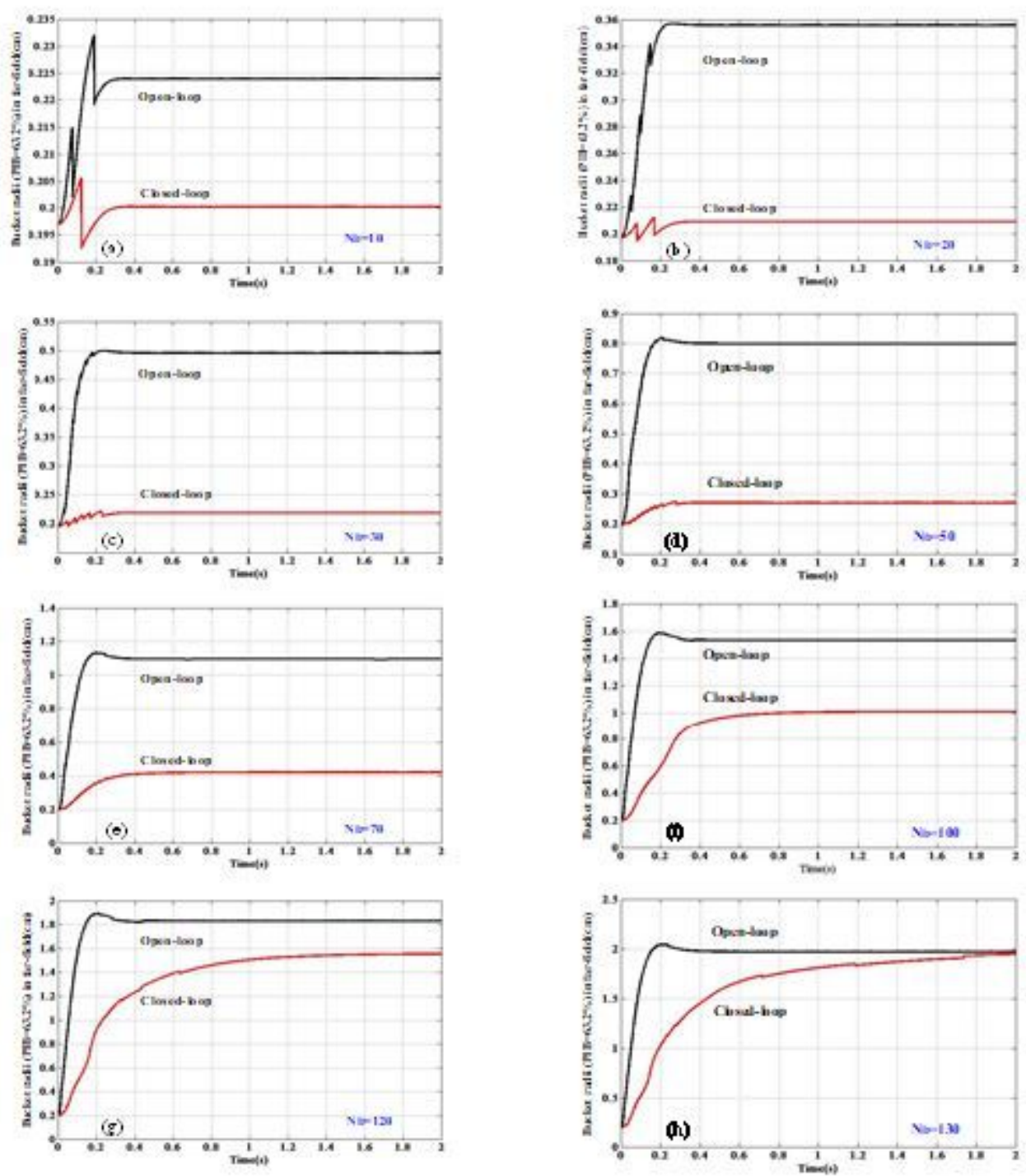

\section{Figure 3}

Comparison of bucket radii (PIB $=63.2 \%$ ) in far-field with different Bradley-Hermann distortion numbers $(\mathrm{ND}=10,20,30,50,70,100,120$ and 130) of open-loop and closed-loop AO systems. 


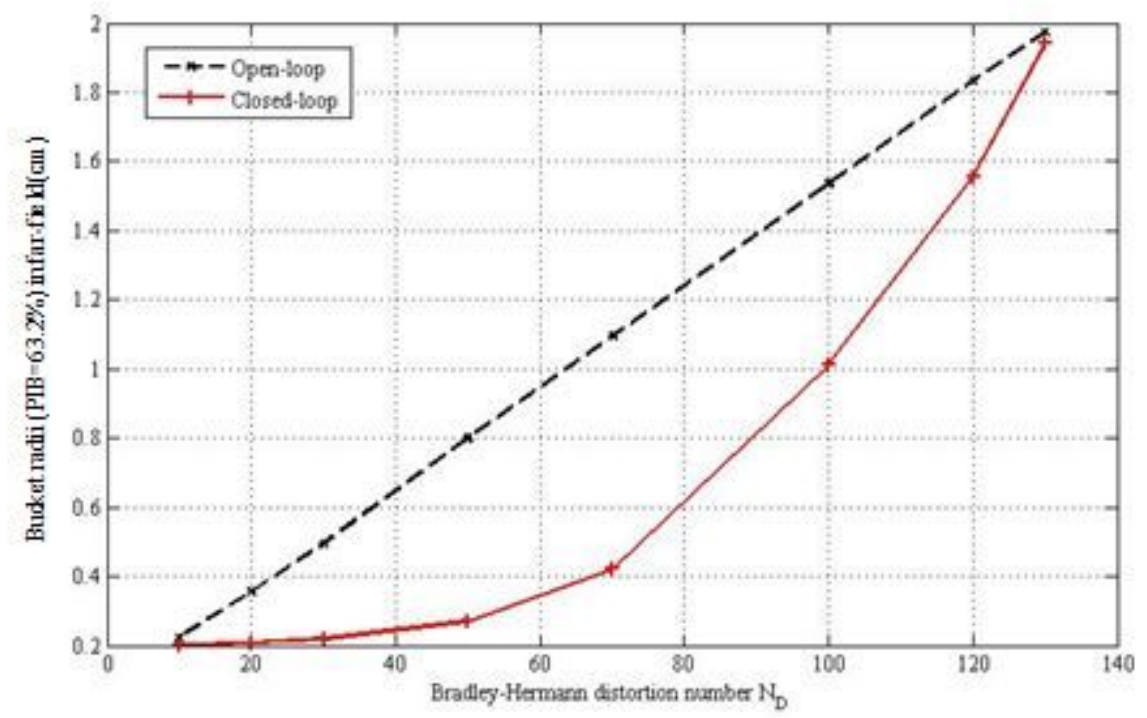

Figure 4

Bucket radii (PIB $=63.2 \%$ ) in far-field with open- and closed-loop operations of the AO system vs. the Bradley-Hermann distortion number (ND = 10, 20,30, 50, 70, 100, 120 and 130)

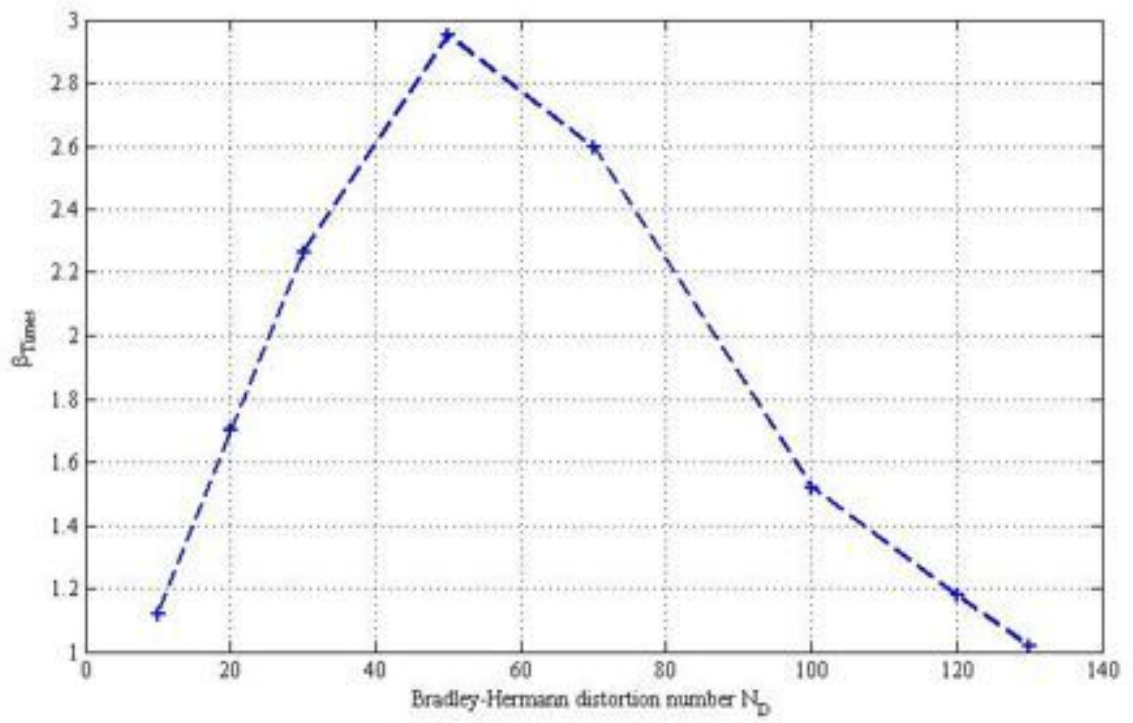

Figure 5

$\beta$ Times vs. ND 


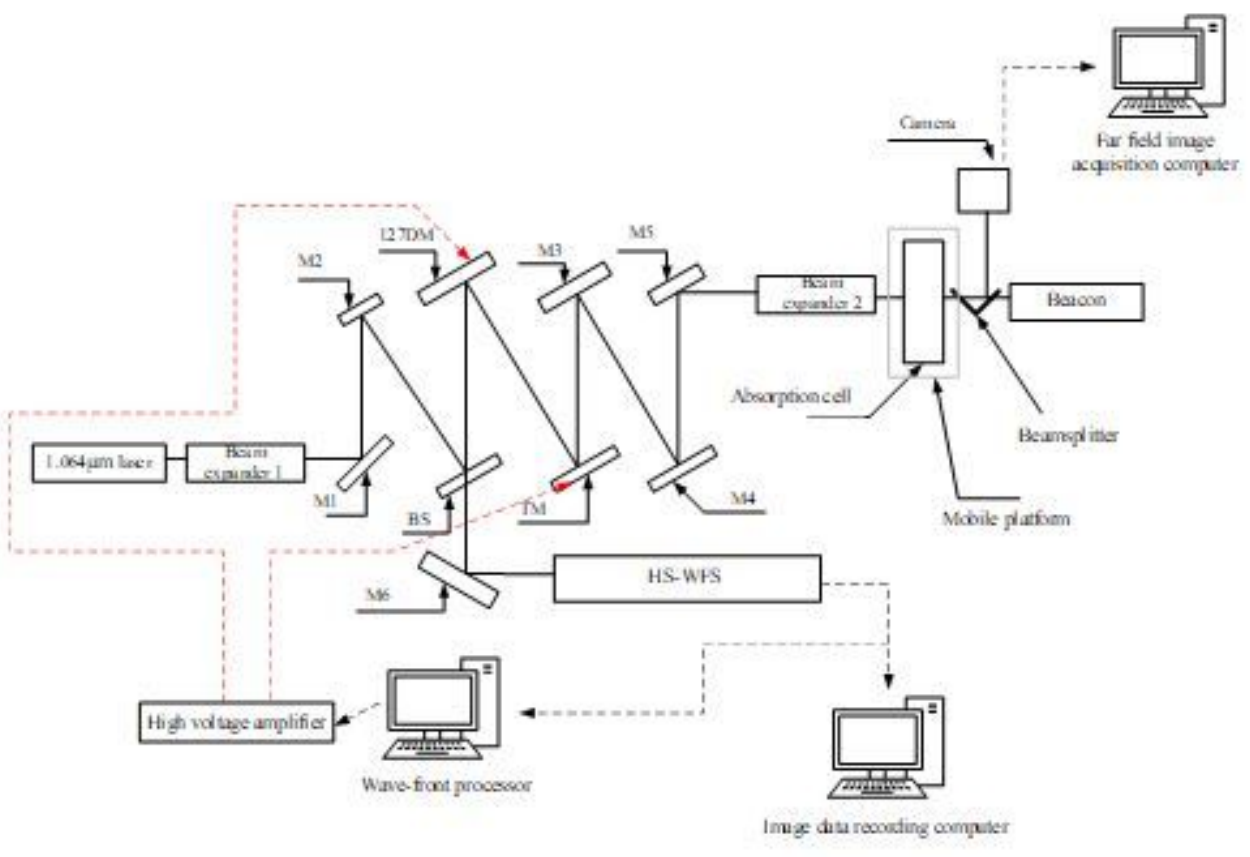

Figure 6

Schematic of experiment with $\mathrm{AO}$ system for phase compensation of thermal blooming.

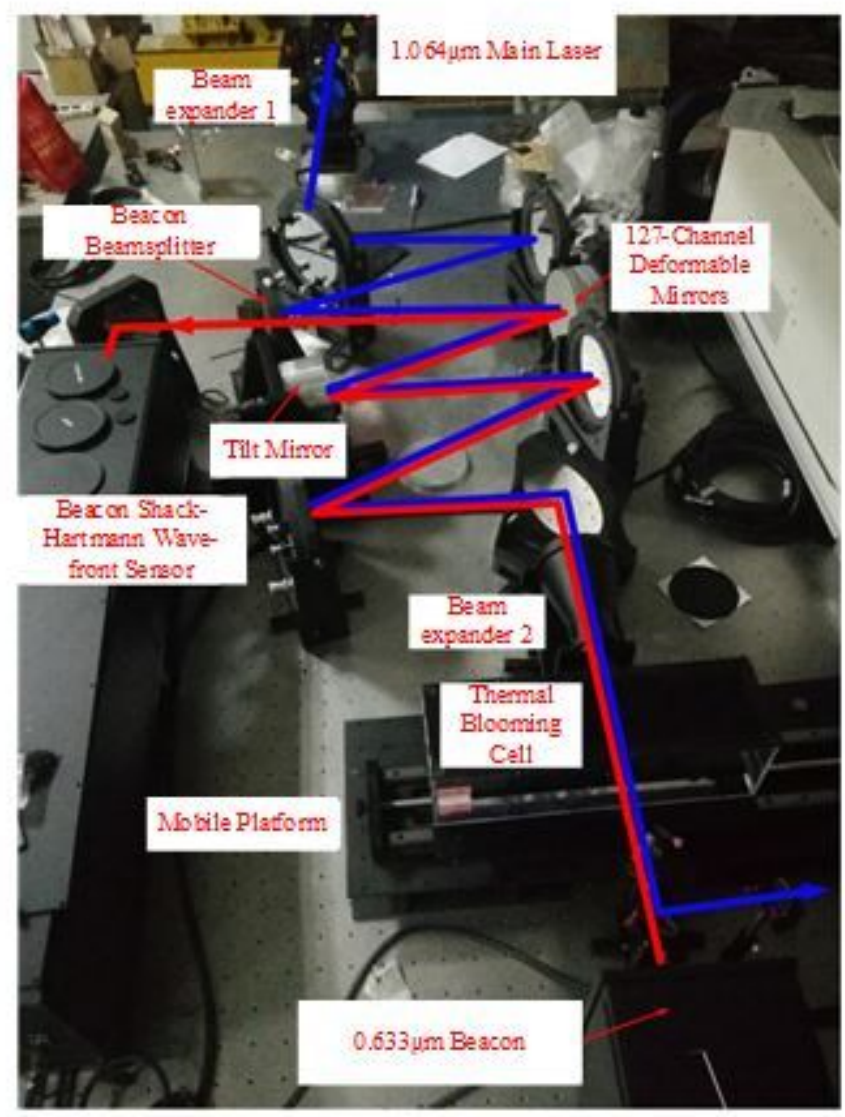

Camera 
Photograph of experimental layout.

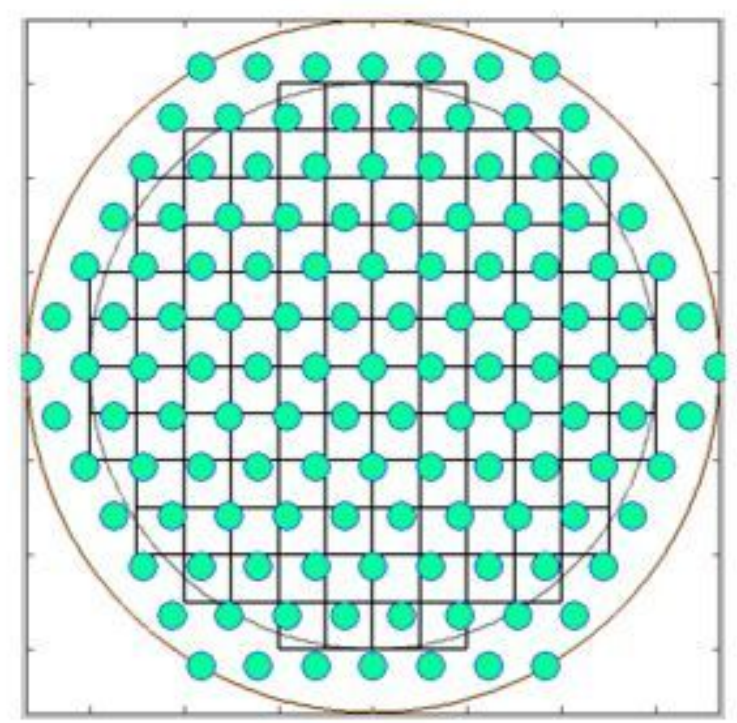

Figure 8

Schematic of deformable mirror with 127 actuators. 

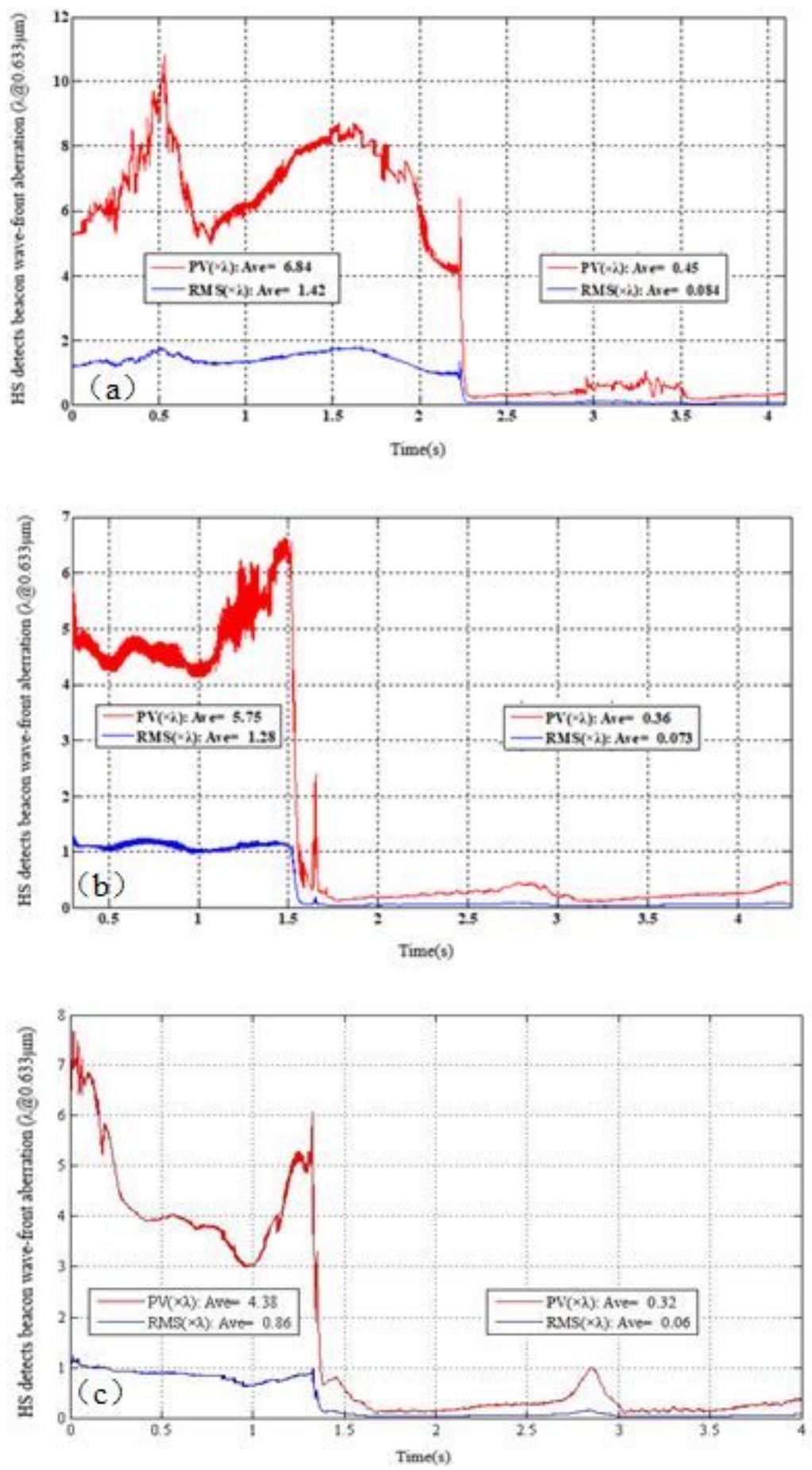

\section{Figure 9}

Comparison of wave-front PV/RMS between open-loop and closed-loop AO systems. In (a) platform velocity $\mathrm{V}$ is $5 \mathrm{~mm} / \mathrm{s}$; wave-front in open-loop $A O$ system, $P V=6.84 \lambda$ and $R M S=1.42 \lambda$; wave-front in closed-loop AO system, $P V=0.45 \lambda$ and RMS $=0.084 \lambda$, in (b) platform moving velocity $V$ is $10 \mathrm{~mm} / \mathrm{s}$; wave-front in open-loop AO system, $P V=5.75 \lambda$ and RMS $=1.28 \lambda$; wave-front in closed-loop AO system, $P V=0.36 \lambda$ and $R M S=0.073 \lambda$ and in (c) platform moving velocity $V$ is $15 \mathrm{~mm} / \mathrm{s}$; wave-front in open-loop 
$\mathrm{AO}$ system, $\mathrm{PV}=4.38 \lambda$ and RMS $=0.86 \lambda$; wave-front in closed-loop $\mathrm{AO}$ system, $\mathrm{PV}=0.32 \lambda$ and $\mathrm{RMS}=$ $0.06 \lambda$.
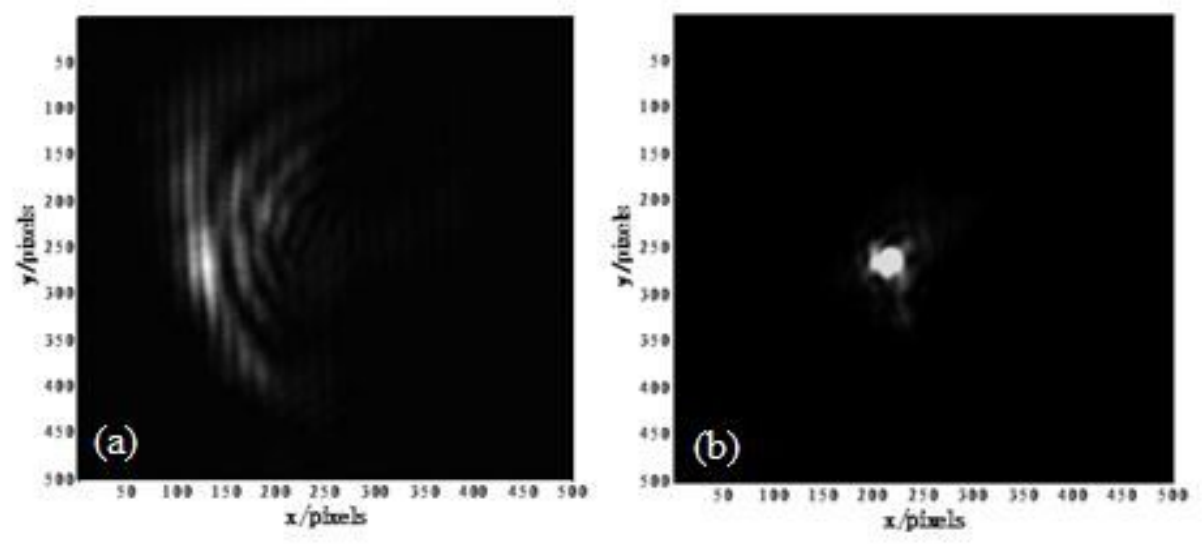

Figure 10

Far-field images of main laser (a) with open-loop operation of the AO system and (b) with closed-loop operation of the AO system. 

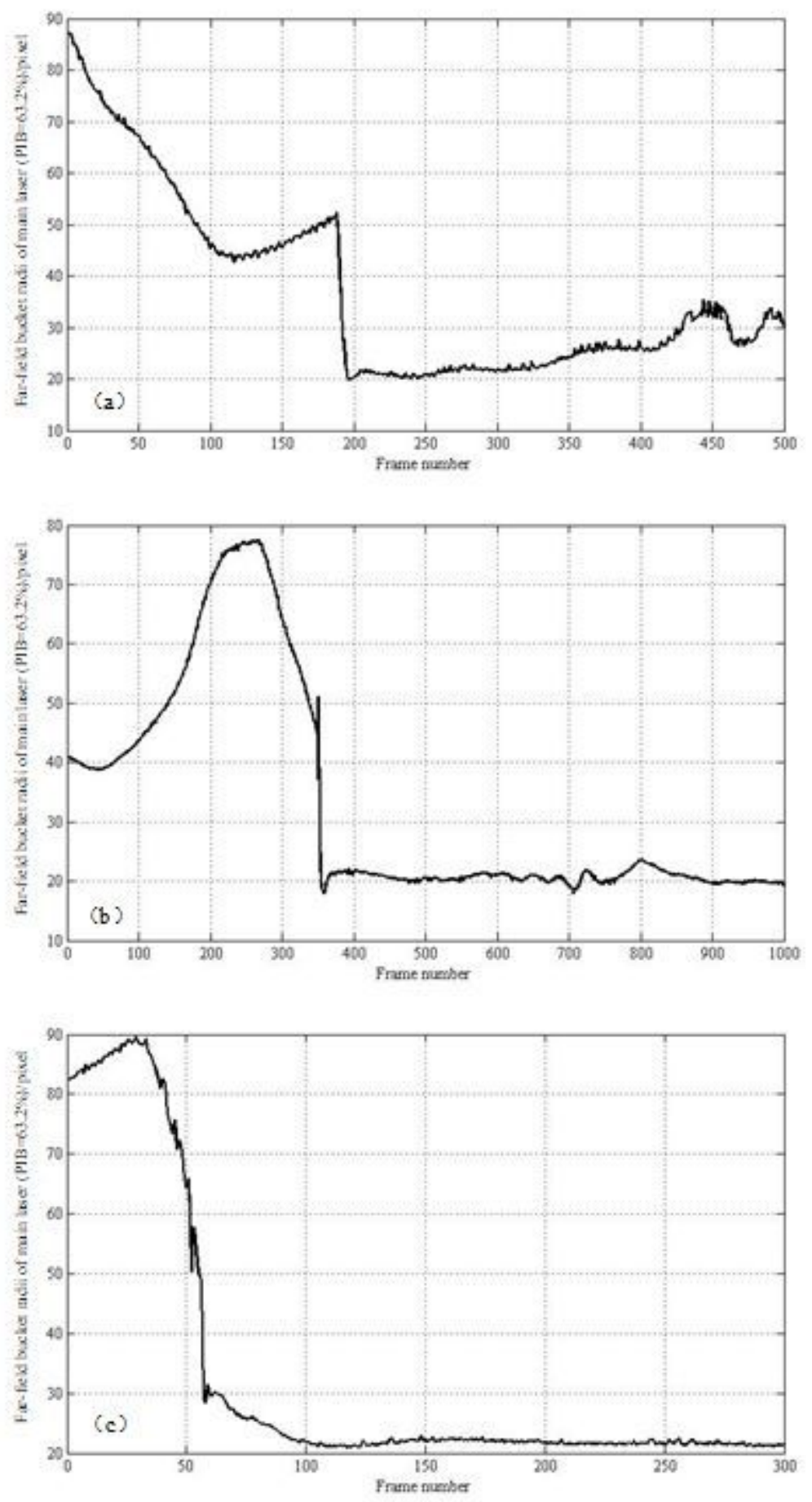

Figure 11

Comparison of far-field bucket radii (PIB $=63.2 \%$ ) between open- and closed-loop operations of $A O$ system. In (a) ND = 82.18, in (b) ND = 74.08 and in (c) ND = 49.27. 


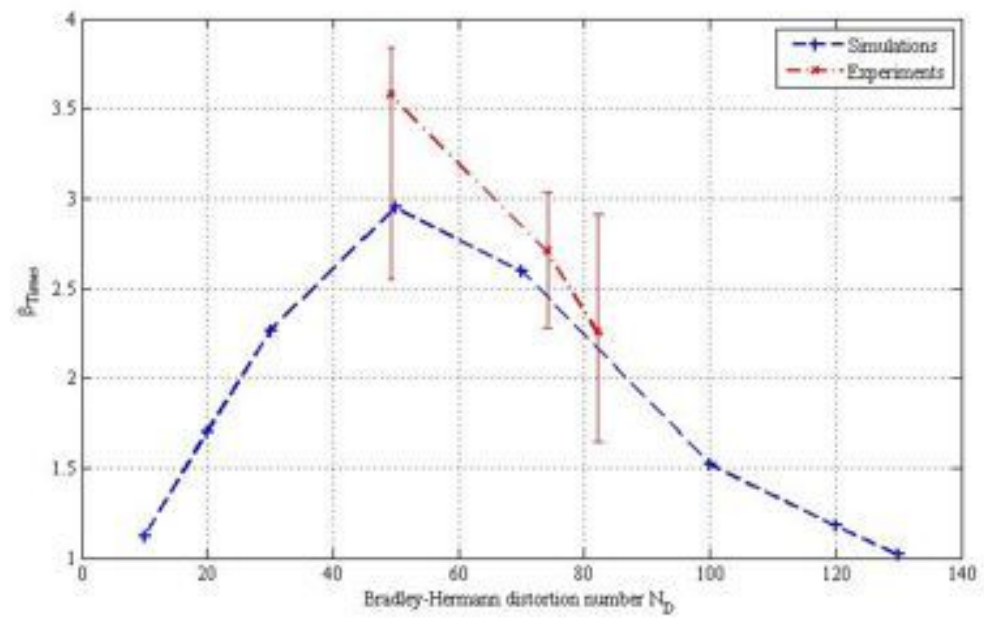

Figure 12

Comparison of $\beta$ Times of simulations with experiments. 